\title{
Signatures of a degenerate many-body state of interlayer excitons in a van der Waals heterostack
}

\author{
Lukas Sigl, ${ }^{1}$ Florian Sigger, ${ }^{1}$ Fabian Kronowetter, ${ }^{1}$ Jonas Kiemle, ${ }^{1}$ Julian Klein $\odot,{ }^{1}$ Kenji Watanabe, ${ }^{2}$ Takashi Taniguchi, ${ }^{2}$ \\ Jonathan J. Finley, ${ }^{1,3}$ Ursula Wurstbauer, ${ }^{1,4, *}$ and Alexander W. Holleitner ${ }^{1,3, \dagger}$ \\ ${ }^{1}$ Walter Schottky Institute and Physics Department, TU Munich, Am Coulombwall 4a, 85748 Garching, Germany \\ ${ }^{2}$ National Institute for Materials Science, Tsukuba, Ibaraki 305-0044, Japan \\ ${ }^{3}$ Munich Center for Quantum Science and Technology (MCQST), Schellingstrasse 4, 80799 München, Germany \\ ${ }^{4}$ Institute of Physics, Westfälische Wilhelms-Universität Münster, Wilhelm-Klemm-Strasse 10, 48149 Münster, Germany
}

(Received 3 March 2020; accepted 7 December 2020; published 22 December 2020)

\begin{abstract}
Atomistic van der Waals heterostacks are ideal systems for high-temperature exciton condensation because of large exciton binding energies and long lifetimes. Charge transport and electron energy-loss spectroscopy showed first evidence of excitonic many-body states in such two-dimensional materials. Pure optical studies, the most obvious way to access the phase diagram of photogenerated excitons, have been elusive. We observe several criticalities in photogenerated exciton ensembles hosted in $\mathrm{MoSe}_{2}-\mathrm{WSe}_{2}$ heterostacks with respect to photoluminescence intensity, linewidth, and temporal coherence pointing towards the transition to a coherent many-body quantum state, consistent with the predicted critical degeneracy temperature. For this state, the estimated occupation is approximately $100 \%$ and the phenomena survive above $10 \mathrm{~K}$.
\end{abstract}

DOI: 10.1103/PhysRevResearch.2.042044

Increasing the interaction strength between quasiparticles can cause strong correlations, collective phenomena, and even the transition to new emergent quantum phases of the many-body state that are substantially different from properties of the weakly interacting system. Many examples exist in condensed matter systems where interacting electrons, spins, and phonons form novel many-body ground states including superconductivity, superfluidity, charge-densitywave phases, polariton condensates, and spin liquids [1-4]. Another predicted many-body state of quasiparticles is a Bose-Einstein condensate of interacting excitons [5-7], that are bound electron-hole pairs in semiconductors. Their many-body phase diagram predicts quantum degeneracy and Bose-Einstein condensation at reduced temperatures and sufficiently high exciton densities [8-12]. In transport experiments, condensed phases of excitons have been reported in quantum Hall bilayer systems for both graphene and coupled semiconductor quantum wells $[13,14]$. The weak exciton binding in these systems, together with the presence of low-energy Goldstone modes, makes it difficult to access the condensates optically and limits the condensation temperature to about $1 \mathrm{~K}$ or lower [5]. Atomically thin, semiconducting monolayers such as transition metal dichalcogenides (TMDCs) exhibit a large exciton binding energy (about $0.5 \mathrm{eV}$ ) [15] and the possibility to form van der Waals

\footnotetext{
*wurstbauer@uni-muenster.de

†holleitner@wsi.tum.de
}

Published by the American Physical Society under the terms of the Creative Commons Attribution 4.0 International license. Further distribution of this work must maintain attribution to the author $(s)$ and the published article's title, journal citation, and DOI. heterostacks [16,17]. In turn, they provide a promising solid state platform for exploring high-temperature exciton condensation and realizing condensate-based applications on a chip $[10,18,19]$. To diminish the limitations, such as short radiative lifetimes [20] and biexcitons [21] present for intralayer excitons in individual monolayers, the spatial overlap of the Coulomb-bound electron-hole pairs can be reduced by placing them in adjacent layers in a double-layer heterostack [22]. Long lifetimes of several tens of nanoseconds, while the binding energies remain substantial $(>0.1 \mathrm{eV})$, have been demonstrated for such photogenerated interlayer excitons [22-24]. Moreover, they act as oriented electric dipoles supporting dipole-dipole interactions and inducing exciton correlations at high densities [18,25-28]. Theoretically, a room-temperature quasicondensation may be possible for these interlayer excitons in TMDC double layers since the maximum condensation temperature is limited by exciton ionization in the high-density regime, and it has been predicted to be a fraction of the exciton binding energy [10]. Nevertheless, the exploration of the many-body phase diagram of photogenerated interlayer excitons in TMDCs including quantum degeneracy and condensation has remained elusive so far.

We present clear signatures of a many-body state of photogenerated excitons in $\mathrm{MoSe}_{2}-\mathrm{WSe}_{2}$ heterostacks at elevated temperatures via several criticalities. The device structure under investigation consists of two rotationally aligned TMDC monolayers [Figs. 1(a) and S1] [23,29]. The cryogenic photoluminescence spectra exhibit only one very sharp emission line (1) at low excitation powers, which dominates all other optical transitions including those arising from intralayer excitons $X_{1 s}^{\mathrm{MoSe}_{2}}, X_{1 s}^{\mathrm{WSe}_{2}}$ and the trions $X_{T}^{\mathrm{MoSe}_{2}}, X_{T}^{\mathrm{WSe}_{2}}$ in the monolayer $\mathrm{MoSe}_{2}$ and $\mathrm{WSe}_{2}$ (Fig. S2) [29]. The emission energy of this state is about $6-7 \mathrm{meV}$ below two further emission peaks named (2) and (3) in Fig. 1(b) that appear 
(a)

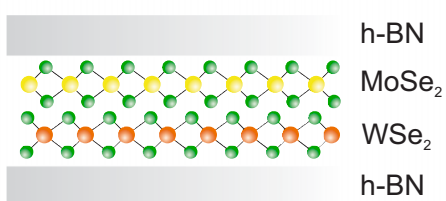

(b)

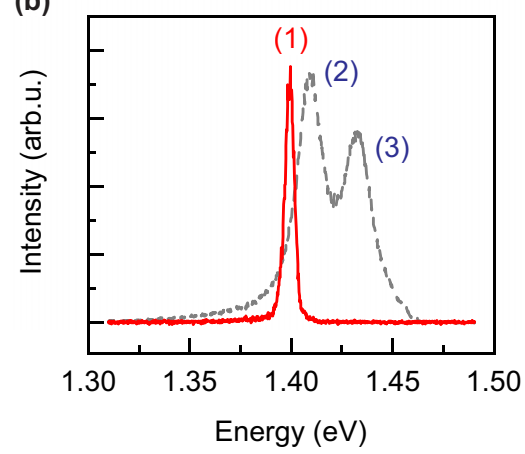

(c)

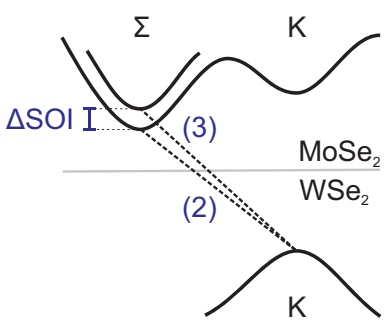

(e)

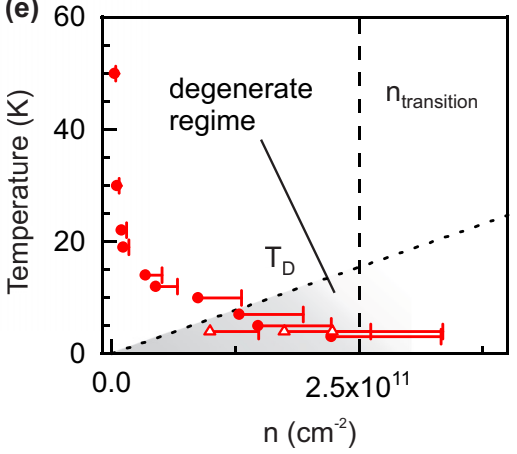

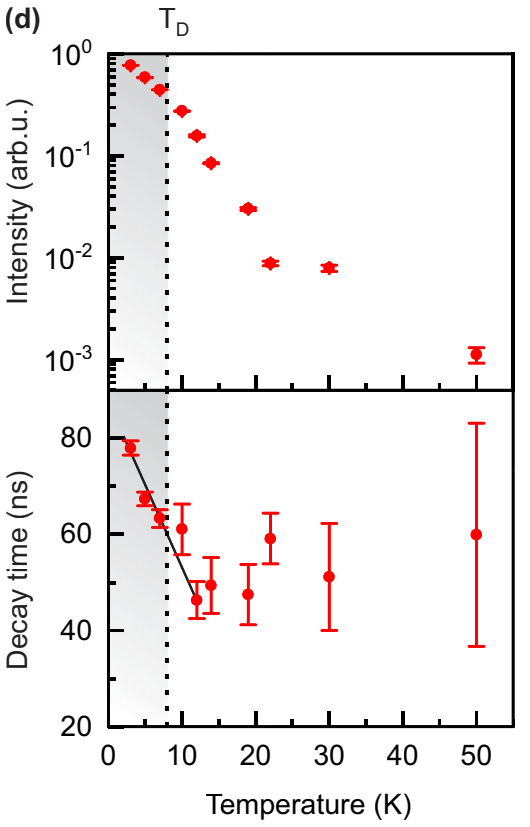

FIG. 1. (a) Schematic of a MoSe $2 / \mathrm{WSe}_{2}$ heterostack encapsulated in hBN. (b) Photoluminescence spectra with a many-body-state-emission peak (1) - as it is observable at a low excitation power of $200 \mathrm{nW}$, and peaks (2) and (3) at $420 \mu \mathrm{W}$ (excitation energy $E_{\text {photon }}=1.946 \mathrm{eV}$ and bath temperature $T=4 \mathrm{~K}$ ). All emission peaks stem from interlayer excitons. (c) Sketch of $\mathrm{MoSe}_{2}$ conduction band and WSe $\mathrm{H}_{2}$ valence band. The dashed lines indicate the momentum indirect interlayer transitions explaining peaks (2) and (3). (d) Intensity (top) and lifetime (bottom) of the many-body state vs bath temperature. The black line is a guide to the eye $\left(E_{\text {photon }}=1.59 \mathrm{eV}\right)$. (e) Bath temperature vs estimated exciton density with the dotted line according to Eq. (1) and $T_{\mathrm{D}}$ the degeneracy temperature. The dashed line is for a transition density $n_{\text {transition }}$ between the regimes where peak (1) dominates and the regime where peaks (2) and (3) occur. Open triangles are data also presented in Fig. 3.

only for higher excitation powers (Fig. S3) [29]. Peaks (2) and (3) have been reported before, both by our group and others $[23,27,30-32]$. We interpret them as arising from reciprocally indirect transitions from spin-orbit-split states at $\Sigma$ in the conduction band $\left(\mathrm{CB}^{\mathrm{MoSe}_{2}}\right)$ of $\mathrm{MoSe}_{2}$ to $K\left(K^{\prime}\right)$ in the valence band $\left(\mathrm{VB}^{\mathrm{WSe}_{2}}\right)$ of $\mathrm{WSe}_{2}$ [Fig. 1(c)], where the photon emission is linked with the emission or absorption of phonons [31]. Intriguingly, the intensity of peak (1) is enhanced by two orders of magnitude at the lowest temperature [Fig. 1(d)]. Since the decay time of this peak also increases by a factor of 2 , the enhanced photon emission suggests that an increasingly dense part of the exciton ensemble emits into the light cone. Notably, this strong enhancement of emission is not observed for peaks (2) and (3) (cf. Fig. S4) [29], and their decay times remain constant in temperature and two orders of magnitude below the one of the emission peak (1). Plotting the diagram of temperature versus estimated exciton density $n$ for peak (1) (see Supplemental Material [29]), we observe that for a temperature below $8 \mathrm{~K}$ [Fig. 1(e)], the excitons are below the expected degeneracy limit obeying $[6,10]$

$$
k_{\mathrm{B}} T_{\mathrm{D}}=2 \pi \hbar^{2} n / m_{X},
$$

with $k_{\mathrm{B}}$ the Boltzmann constant, $T_{\mathrm{D}}$ the degeneracy temperature, $\hbar$ the reduced Planck's constant, and $m_{X}=m_{\mathrm{e}}+m_{\mathrm{h}}$ the effective exciton mass with $m_{\mathrm{e}}=0.54\left(m_{\mathrm{h}}=0.36\right)$ the effective electron (hole) mass [33]. The presented measurements were performed on two devices for temperatures between 3 and $150 \mathrm{~K}$ during several independent cooldowns demonstrating the robustness of the experimentally observed phenomena.
Below $T_{\mathrm{D}}$, almost all interlayer excitons are estimated to emit photons via peak (1) [Fig. 1(b)], i.e., the underlying exciton state exhibits an estimated, relative occupation close to $100 \%$ [Fig. 2(a)]. Figure 2(b) shows that the full width at half maximum (FWHM) of peak (1) reduces to a constant value less than $\sim 5 \mathrm{meV}$ below $T_{\mathrm{D}}$, although the intensity and lifetime still increase [Fig. 1(d)]. Furthermore, we observe a blueshift of peak (1) up to $2 \mathrm{meV}$ (Fig. S5) [29], that is consistent with a repulsive dipole-dipole interaction within the interlayer exciton ensemble $[25,26]$, particularly since they
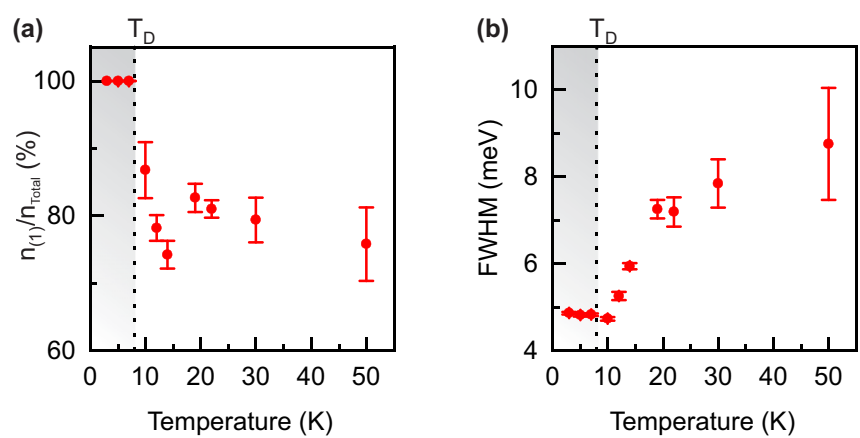

FIG. 2. Temperature dependence and occupation saturation of the many-body state. (a) Relative, estimated occupation of the manybody state with respect to exciton states (2) and (3) vs temperature. (b) Full width at half maximum (FWHM) of the many-body state vs temperature $\left(E_{\text {photon }}=1.59 \mathrm{eV}\right)$. 

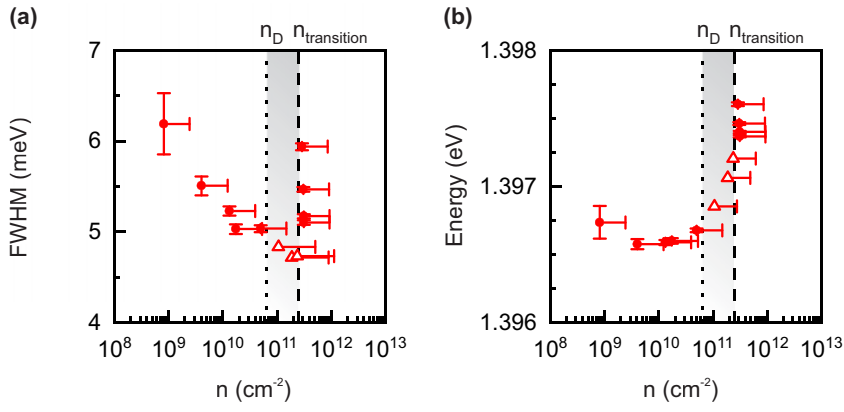

FIG. 3. Density dependence of the many-body state and excitonexciton interaction. (a) Photoluminescence FWHM of the manybody state vs exciton density with the dotted line according to Eq. (1) and $n_{\mathrm{D}}$ the degenerate density at $T=4 \mathrm{~K} . n_{\text {transition }}$ marks the transition to the regime where peaks (2) and (3) occur (dashed line). (b) Emission energy of the many-body state vs exciton density $\left(E_{\text {photon }}=1.59 \mathrm{eV}\right)$. Open triangles are data also presented in Fig. 1(e).

have a longer lifetime and therefore an increasing density at the lowest temperatures [Fig. 1(d) bottom and Fig. 1(e)].

The FWHM of peak (1) decreases as a function of the estimated exciton density, until it reaches a minimum value below $\sim 5 \mathrm{meV}$ in the degenerate regime [triangles in Fig. 3(a)]. For comparison, the dotted line in Fig. 3(a) marks the degeneracy density $n_{\mathrm{D}}$ as described by Eq. (1) for the given temperature, and we added the triangles of Fig. 3(a) into the diagram of Fig. 1(e). The emission energy increases by $\sim 1 \mathrm{meV}$ versus exciton density in the degenerate regime [Fig. 3(b)], which is again consistent with a repulsive dipole-dipole interaction $[25,26]$. For this experiment, the excitation energy is chosen to be $E_{\text {photon }}=1.59 \mathrm{eV}$, which is below the intralayer exciton energies in the $\mathrm{MoSe}_{2}$ and $\mathrm{WSe}_{2}$ monolayers to minimize their impact on the interlayer excitons. The plotted density $n$ only considers interlayer excitons which recombine via peak (1). For an exciton density exceeding $n_{\text {transition }} \sim 2.5 \times 10^{11} \mathrm{~cm}^{-2}$, we observe that the intensity of peak (1) saturates and then peak (2) gradually occurs in the spectra (Fig. S6) [29]. In our point of view, the repulsive dipole-dipole interaction in combination with an excess energy in the exciton ensemble is responsible for the transition at $n_{\text {transition. }}$. Above this critical density, the FWHM of peak (1) rapidly increases [Fig. 3(a)] and the estimated occupation of state (1) is less than $100 \%$ (Fig. S7). [29] The transition cannot arise from a Mott transition, since all emission peaks have long decay times which remain entirely consistent with the photon emission from interlayer excitons (Fig. S8) [29]. For comparison, the expected theoretical value of $n_{\text {Mott }}$ for our heterostack exceeds $10^{12} \mathrm{~cm}^{-2}[10,34]$.

Figure 4(a) shows the temporal coherence visibility measured for emission peak (1) in the degenerate regime below $T_{\mathrm{D}}$ demonstrating that the coherence adheres to the WienerKhinchine theorem [35,36]. In other words, the Fourier transform of the Lorentzian-shaped emission spectra as in Fig. 1(b) overlaps with the experimentally determined coherence visibility with a maximum value of $(88 \pm 7 \%)$. Above $T_{\mathrm{D}}$, the temporal coherence drops significantly [Figs. 4(b) and 4(c)]. Fitting the visibility with exponential decays [black
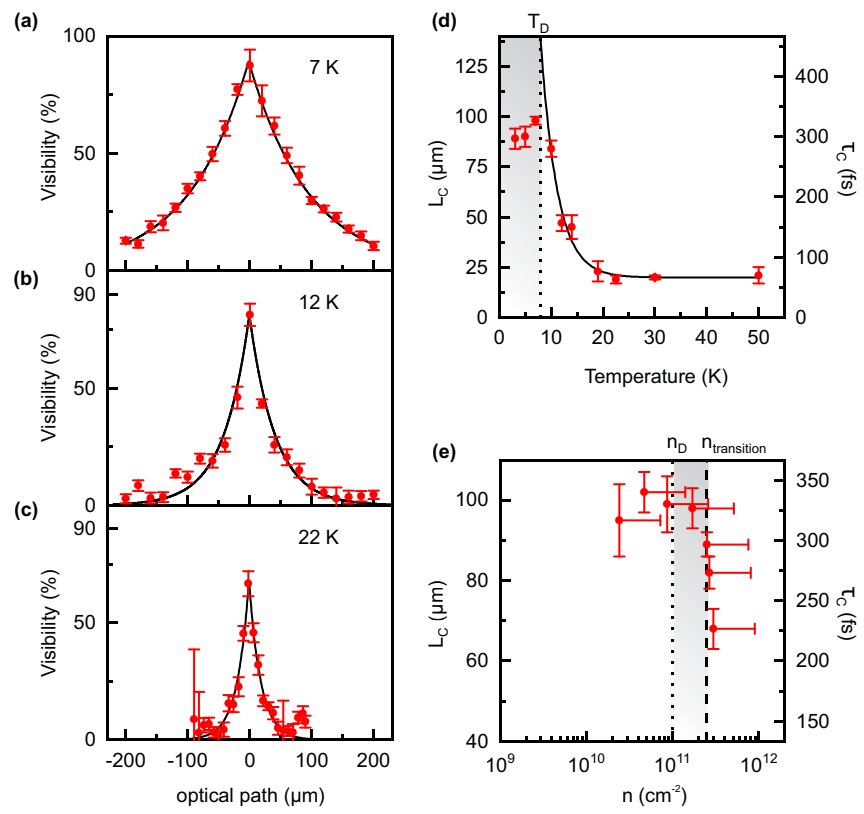

FIG. 4. Fourier-limited temporal coherence in the many-body regime. (a)-(c) Temporal coherence visibility vs optical path for the many-body state for temperatures of 7,12 , and $22 \mathrm{~K}\left(E_{\text {photon }}=\right.$ $1.59 \mathrm{eV})$. Black lines are exponential fits. (d) Temporal coherence length $l_{\mathrm{c}}$ and corresponding coherence time $\tau_{\mathrm{c}}$ for the many-body state vs temperature with an exponential fit above $T_{\mathrm{D}}$. (e) Temporal coherence length vs exciton density in the many-body state at $T=7 \mathrm{~K}$.

lines in Figs. 4(a)-4(c)] reveals a fast drop of the temporal coherence time $\tau_{\mathrm{c}}$ and length $l_{\mathrm{c}}$ [Figs. 4(d) and S9] [29]. Above $T_{\mathrm{D}}, \tau_{\mathrm{c}}$ is most likely limited by thermal broadening of the energy levels as well as thermal activation and interaction with thermally excited acoustic phonons within the twodimensional (2D) materials but also in the adjacent hexagonal boron nitride (hBN). Moreover, the reduced lifetime results in a reduced exciton density and finally an increasing nondegenerate Bose gas that is coexistent for temperatures above $T_{\mathrm{D}}$. Below $T_{\mathrm{D}}$, the optical coherence time is limited to a timescale of $\sim 300 \mathrm{fs}(\sim 14 \mathrm{meV})$, which is approximately five to six orders of magnitude faster than the decay time of peak (1) as depicted in Fig. 4(d). In turn, the Lorentzianshaped emission spectrum of state (1) as in Fig. 1(b) is not radiation limited (Figs. S10 and S11) [29]. Instead, the fast timescale suggests that below $T_{\mathrm{D}}$, the coherence is limited by the coupling to acoustic phonons [longitudinal acoustic (LA), transverse acoustic (TA), or $z$-axis acoustic (ZA)] during the photon emission [37]. The phonon scattering then tentatively has an effect similar to collisional broadening in atom ensembles [38] as the interaction with acoustic phonons gives rise to a phase shift of the emitted photons. This phase shift due to phonon-assisted interband recombination dominates the FWHM of the Lorentzian-shaped lines. The temperature dependence of the interlayer exciton emission energies yields an energy of $E_{\mathrm{p}} \sim 14 \mathrm{meV}$ of phonons interacting with the interlayer excitons (Fig. S12) [29].

The observed energy difference between peaks (2) and (3) $(\sim 25 \mathrm{meV})$ is consistent with the spin-orbit splitting of the $\mathrm{CB}^{\mathrm{MoSe}_{2}}[\Delta \mathrm{SOI}$ in Fig. $1(\mathrm{c})]$ and not with the one in the 
$\mathrm{VB}^{\mathrm{WSe}_{2}}(\sim 450 \mathrm{meV})[33,39]$. A phenomenological polaron fit for the temperature-dependent emission energies below $150 \mathrm{~K}$ gives an exciton-phonon coupling with a Huang-Rhys factor of $S \sim 1.70$ for peak (2) and 1.85 for peak (3) and an average energy of interacting (acoustic) phonons of $E_{\mathrm{p}} \sim 14 \mathrm{meV}$ for both peaks (Fig. S12) [29,31]. Emission peak (1) can be detected only below $50 \mathrm{~K}$ with an emission energy that, unusually, first reduces below and then increases beyond $T_{\mathrm{D}} \sim 8 \mathrm{~K}$ (Fig. S5) [29]. However, as discussed above, its temporal coherence is limited to a timescale of $\sim 300 \mathrm{fs}(\sim 14 \mathrm{meV})$. For comparison, the polaron fit for the direct $1 s$-exciton transition in $\mathrm{MoSe}_{2}\left(\mathrm{WSe}_{2}\right)$ gives $S \sim 2.39(2.28)$ and $E_{\mathrm{p}} \sim 16 \mathrm{meV}$ $(18.7 \mathrm{meV})$. Since the Huang-Rhys factors are different from the ones of the direct transitions at $K\left(K^{\prime}\right)$ and since they interact with phonons, we identify peaks (2) and (3) arising from reciprocally indirect transitions from $\mathrm{SO}$-split states at $\Sigma$ in the $\mathrm{CB}^{\mathrm{MoSe}_{2}}$ to $K\left(K^{\prime}\right)$ in the $\mathrm{VB}^{\mathrm{WSe}_{2}}$ [as sketched in Fig. 1(c)] [31].

We now summarize several arguments which indicate that peak (1) cannot be identified as a single-quasiparticle transition, while peaks (2) and (3) results from exciton recombination in a single-particle framework. On the one hand, peak (1) could correspond to an optical transition from the minima at $K\left(K^{\prime}\right)$ in the $\mathrm{CB}^{\mathrm{MoSe}_{2}}$ to the maxima at $K\left(K^{\prime}\right)$ in the $\mathrm{VB}^{\mathrm{WSe}_{2}}$. However, it is known that this reciprocally direct transition should have a higher energy difference than the indirect one between $\Sigma$ in the $\mathrm{CB}^{\mathrm{MoSe}_{2}}$ to $K\left(K^{\prime}\right)$ in the $\mathrm{VB}^{\mathrm{WSe}_{2}}$ especially at high excess charge carrier densities [22,37,40], which is contrary to our observations. On the other hand, peak (1) could relate to a transition from $\Sigma\left(\mathrm{CB}^{\mathrm{MoSe}_{2}}\right)$ to $\Gamma\left(\mathrm{VB}^{\mathrm{WSe}_{2}}\right)$. However, it is counterintuitive that such a reciprocally indirect state would have a strongly increasing luminescence intensity at lowest temperatures. At the same time, the corresponding phonon density supporting this indirect single-particle transition is known to be small with negligible electron-phonon coupling [41].

Instead, we propose that the emission peak (1) corresponds to a many-body state of interlayer excitons resulting from a repulsive interaction with an energy at the few $\mathrm{meV}$ level, and that this many-body state is intrinsically related to the singleparticle transitions of peaks (2) and (3) [cf. Fig. 1(c)]. Again, two of the most prominent arguments for the interrelation are that peak (1) changes its general behavior as soon as peak (2) appears [dashed lines in Figs. 4(e) and 3], and that the excitonphonon coupling $E_{\mathrm{p}} \sim 14 \mathrm{meV}$ for peaks (2) and (3) coincides with the collision broadening of peak (1) (temporal coherence time $\sim 300 \mathrm{fs}$ ). The interrelation can be further seen in the exciton density dependence of the decay time of peak (2). It starts with a rather long time of $\sim 20$ ns close to $n_{\text {transition }}$, when peak (1) is saturated, and it steadily reduces to the rather short lifetime of peak (3) of $\sim 1 \mathrm{~ns}$ as soon as peak (1) is no longer resolvable at an overall high exciton density (Fig. S8) [29]. Still, the lifetimes of peaks (2) and (3) at high exciton densities are consistent with the photon emission from single-particle interlayer excitons.

The evidence for a many-body state is threefold. First, the state has an estimated relative occupancy of $100 \%$ for experimental conditions such that Eq. (1) is fulfilled. Second, below $T_{\mathrm{D}}$, the intensity of peak (1) is consistent with an increasingly dense exciton population emitting more and more into the light cone. Third, below $T_{\mathrm{D}}$, the FWHM of peak (1) is independent from thermal broadening but it decreases with increasing exciton density, with a limiting temporal coherence of 300 fs. From the polaron fits, we know that up to $\sim 100 \mathrm{~K}$ the energy (coherence time) of the interacting phonons is $E_{\mathrm{p}} \sim 14 \mathrm{meV}$ (300 fs). In turn, for approaching $T_{\mathrm{D}}$ from higher temperatures, we deduce that the exponential increase of the coherence stems from a reduced dephasing of the excitons, as expected for a many-body state approaching the quantum degeneracy [cf. the exponential fit in Fig. 4(d)]. Below $T_{\mathrm{D}}$, the overall emission coherence seems to be limited by the exciton-phonon interaction. Further studies are needed to explore the spatial coherence [42] of the many-body exciton system and the impact of interacting phonons. Peaks (1) and (2) exhibit an energy difference of $\sim 6-7 \mathrm{meV}$. The manybody interaction most likely results from the dipole-dipole interaction $\sim 1-2 \mathrm{meV}$ and possibly by the layer breathing mode as is known for TMDC heterostacks with an energy of $3.7-4.9 \mathrm{meV}\left(30-40 \mathrm{~cm}^{-1}\right)$. Dynamic and static screening effects might further impact the emission energy of peak (1) $[43,44]$. In this picture, the underlying exciton state of peak (2) limits the coherence of peak (1) as soon as the FWHM of peak (1) exceeds the energy difference between the two peaks either by exciton-exciton interactions within the dense ensemble [dashed lines in Figs. 1(e), 3(a), and 4(e)] or by thermal fluctuations [dotted line in Fig. 1(e)]. The FWHM of line (1) of $\sim 5 \mathrm{meV}$ is consistent with the Wiener-Khinchine theorem, i.e., given by the coherence of the emission process (cf. Figs. S10 and S11) [29]. As was put forward by Fogler et al. [10], it is expected that below the degeneracy temperature, the formation of a quantum degenerate Bose gas leads to local exciton superfluidity, where disorder does play a role for the expansion and interaction of the exciton ensemble. Strictly speaking, we cannot exclude possible moiré bands with a flat energy dispersion. However, we do not detect any sign for moiré excitons down to the lowest excitation powers (cf. Fig. S13) [29,45]. Consistently, we detect a homogeneous photoluminescence of peak 1 across the whole heterostack (several micrometers).

In conclusion, we optically generated a high-density ensemble of interlayer excitons in a 2D van der Waals heterostack. In photoluminescence studies, we have observed a threshold behavior on temperature and density, which is consistent with a degenerate many-body exciton state. The observations persist to a temperature between 10 and $20 \mathrm{~K}$. Our results open up opportunities to explore the optical properties of exciton condensates and high-temperature superconductivity in exciton ensembles [10].

We thank R. Schmidt, R. Rappaport, and F. Dubin for discussions. We gratefully acknowledge financial support by the Deutsche Forschungsgemeinschaft (DFG) via Projects No. WU 637/4-1 and No. HO 3324/9-1 and the excellence cluster Munich Center for Quantum Science and Technology (MCQST). J.K. acknowledges support by the Alexander von Humboldt foundation, and K.W. and T.T. from the Elemental Strategy Initiative conducted by the MEXT, Japan and the CREST (JPMJCR15F3), JST. 
[1] J. P. Eisenstein, Exciton condensation in bilayer quantum Hall systems, Annu. Rev. Condens. Matter Phys. 5, 159 (2014).

[2] X. Xi, L. Zhao, Z. Wang, H. Berger, L. Forró, J. Shan, and K. F. Mak, Strongly enhanced charge-density-wave order in monolayer $\mathrm{NbSe}_{2}$, Nat. Nanotechnol. 10, 765 (2015).

[3] A. Banerjee, C. A. Bridges, J.-Q. Yan, A. A. Aczel, L. Li, M. B. Stone, G. E. Granroth, M. D. Lumsden, Y. Yiu, J. Knolle, S. Bhattacharjee, D. L. Kovrizhin, R. Moessner, D. A. Tennant, D. G. Mandrus, and S. E. Nagler, Proximate Kitaev quantum spin liquid behaviour in a honeycomb magnet, Nat. Mater. 15, 733 (2016).

[4] D. Caputo, D. Ballarini, G. Dagvadorj, C. Sánchez Muñoz, M. De Giorgi, L. Dominici, K. West, L. N. Pfeiffer, G. Gigli, F. P. Laussy, M. H. Szymańska, and D. Sanvitto, Topological order and thermal equilibrium in polariton condensates, Nat. Mater. 17, 145 (2018).

[5] D. Snoke, Spontaneous Bose coherence of excitons and polaritons, Science 298, 1368 (2002).

[6] A. Griffin, D. W. Snoke, and S. Stringari, Bose-Einstein Condensation, Revised (Cambridge University Press, Cambridge, UK, 2010).

[7] M. Combescot, R. Combescot, and F. Dubin, Bose-Einstein condensation and indirect excitons: A review, Rep. Prog. Phys. 80, 066501 (2017).

[8] A. A. High, J. R. Leonard, M. Remeika, L. V. Butov, M. Hanson, and A. C. Gossard, Condensation of excitons in a trap, Nano Lett. 12, 2605 (2012).

[9] M. Stern, V. Umansky, and I. Bar-Joseph, Exciton liquid in coupled quantum wells, Science 343, 55 (2014).

[10] M. M. Fogler, L. V. Butov, and K. S. Novoselov, Hightemperature superfluidity with indirect excitons in van der Waals heterostructures, Nat. Commun. 5, 4555 (2014).

[11] K. Cohen, Y. Shilo, K. West, L. Pfeiffer, and R. Rapaport, Dark high density dipolar liquid of excitons, Nano Lett. 16, 3726 (2016).

[12] M. Beian, M. Alloing, R. Anankine, E. Cambril, C. G. Carbonell, A. Lemaître, and F. Dubin, Spectroscopic signatures for the dark Bose-Einstein condensation of spatially indirect excitons, Europhys. Lett. 119, 37004 (2017).

[13] J. P. Eisenstein and A. H. MacDonald, Bose-Einstein condensation of excitons in bilayer electron systems, Nature (London) 432, 691 (2004).

[14] X. Liu, K. Watanabe, T. Taniguchi, B. I. Halperin, and P. Kim, Quantum Hall drag of exciton condensate in graphene, Nat. Phys. 13, 746 (2017).

[15] A. Chernikov, T. C. Berkelbach, H. M. Hill, A. Rigosi, Y. Li, O. B. Aslan, D. R. Reichman, M. S. Hybertsen, and T. F. Heinz, Exciton Binding Energy and Nonhydrogenic Rydberg Series in Monolayer $\mathrm{WS}_{2}$, Phys. Rev. Lett. 113, 076802 (2014).

[16] F. Withers, O. D. Pozo-Zamudio, A. Mishchenko, A. P. Rooney, A. Gholinia, K. Watanabe, T. Taniguchi, S. J. Haigh, A. K. Geim, A. I. Tartakovskii, and K. S. Novoselov, Light-emitting diodes by band-structure engineering in van der Waals heterostructures, Nat. Mater. 14, 301 (2015).

[17] K. S. Novoselov, A. Mishchenko, A. Carvalho, and A. H. C. Neto, 2D materials and van der Waals heterostructures, Science 353, aac9439 (2016).

[18] Z. Wang, D. A. Rhodes, K. Watanabe, T. Taniguchi, J. C. Hone, J. Shan, and K. F. Mak, Evidence of high-temperature exciton condensation in two-dimensional atomic double layers, Nature (London) 574, 76 (2019).

[19] A. Kogar, M. S. Rak, S. Vig, A. A. Husain, F. Flicker, Y. I. Joe, L. Venema, G. J. MacDougall, T. C. Chiang, E. Fradkin, J. van Wezel, and P. Abbamonte, Signatures of exciton condensation in a transition metal dichalcogenide, Science 358, 1314 (2017).

[20] G. Moody, C. Kavir Dass, K. Hao, C.-H. Chen, L.-J. Li, A. Singh, K. Tran, G. Clark, X. Xu, G. Berghäuser, E. Malic, A. Knorr, and X. Li, Intrinsic homogeneous linewidth and broadening mechanisms of excitons in monolayer transition metal dichalcogenides, Nat. Commun. 6, 8315 (2015).

[21] T. Mueller and E. Malic, Exciton physics and device application of two-dimensional transition metal dichalcogenide semiconductors, npj 2D Mater. Appl. 2, 29 (2018).

[22] P. Rivera, J. R. Schaibley, A. M. Jones, J. S. Ross, S. Wu, G. Aivazian, P. Klement, K. Seyler, G. Clark, N. J. Ghimire, J. Yan, D. G. Mandrus, W. Yao, and X. Xu, Observation of long-lived interlayer excitons in monolayer $\mathrm{MoSe}_{2}-\mathrm{WSe}_{2}$ heterostructures, Nat. Commun. 6, 6242 (2015).

[23] B. Miller, A. Steinhoff, B. Pano, J. Klein, F. Jahnke, A Holleitner, and U. Wurstbauer, Long-lived direct and indirect interlayer excitons in van der Waals heterostructures, Nano Lett. 17, 5229 (2017).

[24] P. Merkl, F. Mooshammer, P. Steinleitner, A. Girnghuber, K.-Q. Lin, P. Nagler, J. Holler, C. Schüller, J. M. Lupton, T. Korn, S. Ovesen, S. Brem, E. Malic, and R. Huber, Ultrafast transition between exciton phases in van der Waals heterostructures, Nat. Mater. 18, 691 (2019).

[25] G. J. Schinner, J. Repp, E. Schubert, A. K. Rai, D. Reuter, A. D. Wieck, A. O. Govorov, A. W. Holleitner, and J. P. Kotthaus, Confinement and Interaction of Single Indirect Excitons in a Voltage-Controlled Trap Formed Inside Double InGaAs Quantum Wells, Phys. Rev. Lett. 110, 127403 (2013).

[26] J. Kiemle, F. Sigger, M. Lorke, B. Miller, K. Watanabe, T. Taniguchi, A. Holleitner, and U. Wurstbauer, Control of the orbital character of indirect excitons in $\mathrm{MoS}_{2} / \mathrm{WS}_{2}$ heterobilayers, Phys. Rev. B 101, 121404(R) (2020).

[27] L. A. Jauregui, A. Y. Joe, K. Pistunova, D. S. Wild, A. A. High, Y. Zhou, G. Scuri, K. D. Greve, A. Sushko, C.-H. Yu, T. Taniguchi, K. Watanabe, D. J. Needleman, M. D. Lukin, H. Park, and P. Kim, Electrical control of interlayer exciton dynamics in atomically thin heterostructures, Science 366, 870 (2019).

[28] A. Ciarrocchi, D. Unuchek, A. Avsar, K. Watanabe, T. Taniguchi, and A. Kis, Polarization switching and electrical control of interlayer excitons in two-dimensional van der Waals heterostructures, Nat. Photonics 13, 131 (2019).

[29] See Supplemental Material at http://link.aps.org/supplemental/ 10.1103/PhysRevResearch.2.042044 for materials preparation and details, estimation of exciton density and occupation numbers, measurement details on photoluminescence spectra and lifetimes of all inter- and intralayer excitons, details on temporal coherence experiments, control experiments on second sample, as well as polaron fit of interlayer excitons.

[30] J. Kunstmann, F. Mooshammer, P. Nagler, A. Chaves, F. Stein, N. Paradiso, G. Plechinger, C. Strunk, C. Schüller, G. Seifert, D. R. Reichman, and T. Korn, Momentum-space indirect interlayer excitons in transition-metal dichalcogenide van der Waals heterostructures, Nat. Phys. 14, 801 (2018). 
[31] A. T. Hanbicki, H.-J. Chuang, M. R. Rosenberger, C. S. Hellberg, S. V. Sivaram, K. M. McCreary, I. I. Mazin, and B. T. Jonker, Double indirect interlayer exciton in a $\mathrm{MoSe}_{2} / \mathrm{WSe}_{2}$ van der Waals heterostructure, ACS Nano 12, 4719 (2018).

[32] D. Unuchek, A. Ciarrocchi, A. Avsar, Z. Sun, K. Watanabe, T. Taniguchi, and A. Kis, Valley-polarized exciton currents in a van der Waals heterostructure, Nat. Nanotechnol. 14, 1104 (2019).

[33] A. Kormányos, G. Burkard, M. Gmitra, J. Fabian, V. Zólyomi, N. D. Drummond, and V. Fal'ko, k $\cdot \mathbf{p}$ theory for twodimensional transition metal dichalcogenide semiconductors, 2D Mater. 2, 022001 (2015).

[34] J. Wang, J. Ardelean, Y. Bai, A. Steinhoff, M. Florian, F. Jahnke, X. Xu, M. Kira, J. Hone, and X.-Y. Zhu, Optical generation of high carrier densities in 2D semiconductor heterobilayers, Sci. Adv. 5, eaax0145 (2019).

[35] J. W. Goodman, Statistical Optics, Wiley Classics Library (Wiley, New York, 1985).

[36] Time Series and Statistics, edited by J. Eatwell, M. Milgate, and P. Newman (Palgrave Macmillan, London, 1990).

[37] J. Klein, A. Kuc, A. Nolinder, M. Altzschner, J. Wierzbowski, F. Sigger, F. Kreupl, J. J. Finley, U. Wurstbauer, A. W. Holleitner, and M. Kaniber, Robust valley polarization of helium ion modified atomically thin $\mathrm{MoS}_{2}$, 2D Mater. 5, 011007 (2017).

[38] R. Loudon, The Quantum Theory of Light, 3rd ed. (Oxford University Press, Oxford, UK, 2000).
[39] K. F. Mak, K. He, J. Shan, and T. F. Heinz, Control of valley polarization in monolayer $\mathrm{MoS}_{2}$ by optical helicity, Nat. Nanotechnol. 7, 494 (2012).

[40] A. Steinhoff, M. Rösner, F. Jahnke, T. O. Wehling, and C. Gies, Influence of excited carriers on the optical and electronic properties of $\mathrm{MoS}_{2}$, Nano Lett. 14, 3743 (2014).

[41] Y. Ge and A. Y. Liu, Phonon-mediated superconductivity in electron-doped single-layer $\mathrm{MoS}_{2}$ : A first-principles prediction, Phys. Rev. B 87, 241408(R) (2013).

[42] J. Repp, G. J. Schinner, E. Schubert, A. K. Rai, D. Reuter, A. D. Wieck, U. Wurstbauer, J. P. Kotthaus, and A. W. Holleitner, Confocal shift interferometry of coherent emission from trapped dipolar excitons, Appl. Phys. Lett. 105, 241101 (2014).

[43] S. Dietl, S. Wang, D. Schuh, W. Wegscheider, J. P. Kotthaus, A. Pinczuk, A. W. Holleitner, and U. Wurstbauer, Collective electronic excitation in a trapped ensemble of photogenerated dipolar excitons and free holes revealed by inelastic light scattering, Phys. Rev. B 95, 085312 (2017).

[44] M. Florian, M. Hartmann, A. Steinhoff, J. Klein, A. W. Holleitner, J. J. Finley, T. O. Wehling, M. Kaniber, and C. Gies, The dielectric impact of layer distances on exciton and trion binding energies in van der Waals heterostructures, Nano Lett. 18, 2725 (2018).

[45] K. L. Seyler, P. Rivera, H. Yu, N. P. Wilson, E. L. Ray, D. G. Mandrus, J. Yan, W. Yao, and X. Xu, Signatures of moirétrapped valley excitons in $\mathrm{MoSe}_{2} / \mathrm{WSe}_{2}$ heterobilayers, Nature (London) 567, 66 (2019). 